\title{
INSTRUMENTO DE CONSULTA DE ENFERMAGEM A PESSOAS COM HIPERTENSÃO ARTERIAL EM TRATAMENTO AMBULATORIAL.
}

\author{
Angela Maria Geraldo Pierin* \\ Marcia Regina Car*
}

PIERIN, A.M.G.; CAR, M.R. Instrumento de consulta de enfermagem a pessoas com hipertensāo arterial em tratamento ambula torial. Rev. Esc. USP, v. 26, n. 1, p. 17-32, mar. 1992.

$O$ artigo enfatiza a importância da atuação do enfermeiro junto a pessoas hipertensas, apresentando um modelo de impresso de consulta de enfermagem utilizado a nivel ambulatorial.

UNITERMOS: Consulta de enfermagem, Hipertensão.

\section{INTRODUÇÃO}

A consulta de enfermagem, atividade específica do enfermeiro, tem sido utilizada como uma forma de assistência à pessoa com hipertensão arterial, considerando a promoção de condiçōes favoráveis para o tratamento e o direcionamento para o auto cuidado.

Um dos maiores desafios para o hipertenso é aceitar a convivência com a cronicidade da doença; a aceitação da doença não é tarefa fácil e se associa com sentimentos de tristeza, raiva, agressividade e hostilidade, cuja superação se faz através da conscientização de sua condição e necessidade de enfrentar a moléstia, com o seguimento do tratamento proposto, para que possa levar uma vida o mais próximo do normal. Este processo de adaptação exige da pessoa com afecção crônica, dentre outros, conhecimento acerca da doença, manifestações, sinais e sintomas e muita vontade de cooperar ativamente no seu tratamento.

A hipertensão arterial é uma doença de alta prevalência em nosso meio, atingindo cerca de 10 a $20 \%$ da população adulta jovem, sendo que $90 \%$ dos hipertensos possuem a chamada hipertensão arterial essencial ou primária, cuja causa básica é desconhecida. Quando não tratada acarreta uma série de distúrbios orgânicos comprometendo órgãos vitais como o coração, cérebro e rins. Estudos apontam a correlação do acidente vascular cerebral, insuficiência coronariana e renal, bem como fenômenos ateroscleróticos como conseqüêntes de hiper

\footnotetext{
- Enfermeira. Mestre em Enfermagem. Assistente do Departamento de Enfermagem Médico-Cirúrgico, disciplina de Enfermagem Médico-Cirúrgica, EEUSP.
} 
tensão arterial severa ${ }^{3}$. No Brasil o grupo de doenças cardiovasculares constitui a primeira causa de mortalidade, sendo responsável, em 1984, por cerca de $25 \%$ dos óbitos ocorridos, sendo neste grupo de doenças o acidente vascular e a doença cardíaca isquêmica os mais incidentes'.

Atualmente dispõe-se de uma diversidade de medicamentos anti-hipertensivos, com ação eficiente no controle dos níveis tensionais, porém verifica-se que isto nem sempre é conseguido. A dificuldade em controlar a pressão arterial é um fato freqüentemente observado no assistência junto a grupos de pessoas hipertensas, fazendo-se presente principalmente nos assintomáticos, que, muitas vezes, na vigência do tratamento, passam a ter sintomas indesejáveis advindos dos efeitos colaterais dos medicamentos. Vários estudos apontam a não aderência do hipertenso ao tratamento, apresentando índices que oscilam de 30 a $50 \%$. Enquanto que outros enfatizam a importância do papel do enfermeiro junto a esta clientela, aumentando a aderência e persistência ao tratamento'. Considera-se, portanto, inegável a necessidade de articulação entre os membros da equipe de saúde voltada à problemática do doente hipertenso.

Inúmeros aspectos influenciam a observância do tratamento, tais como situação sócio-econômica, grau de instrução, idade, atividades que executa, sentimentos e conhecimentos sobre a doença, crenças de saúde, estilo de vida, experiência anterior com a doença no meio em que vive, percepção da seriedade do problema, complexidade do tratamento, atitudes decorrentes do sistema de saúde vigente, efeitos colaterais dos medicamentos, percepção social do problema e relacionamento inadequado com membros da equipe de saúde.

Considera-se que a educação para a saúde dos hipertensos é ponto relevante para obtenção de êxito no controle da pressão arterial elevada; reforçando-se também a necessidade do direcionamento para o auto cuidado, no qual a enfermeira deve atuar como elemento central diretivo deste processo.

Condutas específicas de enfermagem têm sido adotadas com a finalidade de se obter maior controle e adesão do hipertenso ao tratamento, destacando-se, dentre elas, a consulta de enfermagem. PAVANI et al ${ }^{6}$ enfatizaram os principais aspectos desta estratégia: possibilidade de uma análise global da situação do doente a partir do caráter individual da consulta de enfermagem, obtenção de dados permitindo maior conhecimento da realidade do hipertenso, considerando os fatores ambientais associados às condiçōes físicas e psicológicas; além de uma busca contínua de conhecimentos e crescimento profissional.

A partir dessas considerações e com o objetivo de promover o hipertenso para o auto cuidado, tendo em vista o controle da pressão arterial e aderência ao tratamento, a consulta de enfermagem tem sido utilizada em um serviço ambulatorial que atende exclusivamente pessoas hipertensas, como uma estratégia para a assistência de enfermagem.

\section{CARACTERÍSTICAS DO INSTRUMENTO DA CONSULTA DE ENFERMAGEM}

O instrumento utilizado (Anexo I) compõe-se de seis partes distintas:

I - Identificação do cliente

II - Entrevista 


\section{III - Exame Físico e dados pessoais \\ IV - Listagem de problemas \\ $V$ - Prescrição de enfermagem \\ VI - Evolução de enfermagem}

Na parte de identificação são considerados dados bio-sociais relevantes para a caracterização e individualização da clientela, bem como a interação destes com o processo de aderência ao tratamento. Quanto ao sexo, apesar de estudos apontarem maior prevalência para o feminino, principalmente após 50 anos, há evidências também de uma distribuição eqüitativa da hipertensão arterial para os dois sexos's. Porém os homens são em geral menos obedientes do que as mulheres no cumprimento da prescrição ${ }^{9}$. Já no que se relaciona à idade e escolaridade, estudo realizado na Liga de Diagnóstico e Tratamento da Hipertensão Arterial, apontou serem mais propensos ao abandono os homens com menos de $\mathbf{4 0}$ anos e os analfabetos, não se encontrando correlação entre raça e estado civil ${ }^{4}$. A ocupação também merece ser analisada, não só considerando a inserção do hipertenso dentro do contexto de uma atividade ocupacional, bem como as suas implicaçōes sociais, através de mecanismos desencadeadores e facilitadores da elevação dos níveis tensionais.

Na parte da entrevista os pontos principais a serem levantados dizem respeito ao conhecimento da sua problemática de saúde, da hipertensão arterial, do tratamento medicamentoso e dietético, por terem fundamental importância na avaliação não só do grau de conhecimento sobre a doença e tratamento, bem como a sua percepção quanto à seriedade do problema. A influência de hábitos culturais e crenças de saúde é inegável neste aspecto ${ }^{2}$. Os efeitos colaterais dos medicamentos, ocasionando sintomatologia desagradável, e o seu custo também são fatores que contribuem para a não aderência do hipertenso ao tratamento.

Os dados relacionados à alimentação, seja no que se refere à quantidade de sal e composição calórica, fazem parte do roteiro de entrevista da consulta de enfermagem. A obesidade, apesar de ter papel secundário na gênese da hipertensão arterial, interfere diretamente nos níveis tensionais, Já quanto ao sal presente na dieta, vários estudos apontam correlação do íon sódio com a hipertensão arterial, se bem que dentre os hipertensos existam aqueles resistentes e os sensíveis a este componente da dieta, mas há concordância sensível de que a restrição do sal contribui no controle da pressão arterials.

Hábitos como fumo e a ingestão de bebida alcóolica também merecem avaliação, o primeiro por apresentar correlação estreita com a arterioesclerose, que seria um ponto agravante da hipertensão arterial, e o segundo, também como um fator de risco, sendo a sua abolição ou redução recomendada.

A ingestão de hormônios anticoncepcionais pelas mulheres é apontada como fator desencadeante e até como causa da hipertensão arterial, portanto merecendo avaliação por parte da enfermeira.

A caracterização da atividade física rotineira visa coletar dados que forneçam subsídios para a adequaçāo deste hábito seja na restrição de exercícios físicos exaustivos ou na adequação de uma atividade regular que propície a redução da pressão arterial, tais como os exercícios físicos aeróbicos como o caminhar regularmente e andar de bicicleta, sempre considerando a frequiência, duração c 
intensidade dos mesmos. Nesta área o ideal é contar com a participação de um profissional especializado, para avaliação e implementação da atividade física, principalmente nos pacientes obesos.

A III parte do instrumento, que compreende exame físico e dados pessoais, inicia-se com registro dos parâmetros vitais como o pulso, apical e periférico e a pressão arterial na posição supina e em pé, segundo técnica preconizada (Anexo II). Dados antropométricos como peso e altura visam avaliar principalmente a presença de obesidade e a medida da circunferência branquial para a adequação da largura do mânguito a ser utilizado. A seguir serão avaliados os seguimentos corporais na seguinte ordem:

\section{Cabeça e pescoço:}

a) boca: condição dentária, mucosa oral, cavum, presença de sialosquese ou outras alterações;

b) olhos: integridade, alterações visuais (escotomas, secreção), uso de lentes corretivas e consulta periódica ao oftalmologista; na presença de edema palpebral verificar periodicidade;

c) ouvidos: integridade, acuidade, presença de alterações como dor, zumbido, secreção;

d) nariz: integridade, presença de alterações como epistaxe e congestão;

e) pescoço: integridade e presença de alterações como extase jugular;

f) demais sintomas específicos: verificar a presença de cefaléia, tontura, edema facial e outros que o paciente possa relatar.

2. Tronco e abdômen, avaliando:

a) integridade, coloração e turgor do tegumento cutâneo;

b) mama: investigar a realização periódica do auto-exame e, na palpação, alterações específicas como a presença de nódulos, coloração alterada e galactorréia;

c) alteraçōes cárdio-respiratórias: pré cardialgia, palpitações, dispnéia (caracterizar frequiência, intensidade, duração, tipo e correlação com fatores desencadeantes), tosse (intensidade e qualidade);

d) outros: no caso de dor lombar associar com atividade ocupacional e exercícios físicos.

3. Membros superiores e inferiores, avaliar:

a) integridade, coloração e turgor do revestimento cutâneo;

b) presença de edema, varizes, câimbras, dor muscular;

c) alterações na locomoção, sensibilidade e movimentação;

d) outras alterações não identificadas nestes segmentos corporais. 
4. Sistema reprodutor, investigar:

a) alterações menstruais como dismenorréia, menopausa;

b) alteraçōes vaginais como corrimento, prurido;

c) alterações na vida sexual incluindo frigidez, impotência, diminuição da libido;

d) realização periódica de Papanicolau;

e) uso de métodos contraceptivos.

5. Imunização

Verificar esquema de imunização para adulto: vacina anti-tetânica e realização de PPD.

6. Queixas

a) de ordem física: verificar a existência de outras alterações não identificadas na entrevista e exame físico;

b) de ordem social: condições de trabalho, renda familiar e individual, compra de medicamentos, moradia (aluguel, condições de saneamento básico), posição que ocupa na família;

c) de ordem emocional: estabilidade psíquica frente a situações estressantes;

d) de ordem espiritual: influência de crenças religiosas.

\section{Dados complementares}

Identificar resultados de exames laboratoriais recentes (sódio, potássio, glicemia, urina I, triglicérides, colesterol), eletrocardiograma, urografia excretora.

O mapa de problemas que compõe a parte IV do impresso será formado pelos problemas identificados na entrevista e exame físico; a partir de então serão traçadas condutas específicas de enfermagem, constituindo-se dessa forma a prescrição de enfermagem (Parte V), que deverá priorizar os problemas. A cada problema levantado corresponderá uma ou mais condutas de enfermagem que deverão se basear principalmente na orientação para o auto cuidado. Os problemas não trabalhados em uma primeira consulta, deverão automaticamente ser considerados nos retornos subseqüentes.

A evolução de enfermagem (Parte VI) será efetuada em todos os retornos dos clientes ao serviço, onde serão verificados dados vitais e antropométricos (pulso, pressão arterial, peso), investigação da resolução ou não dos problemas levantados anteriormente, relação de novos problemas e o estabelecimento de novas condutas de enfermagem e reforço daquelas já emitidas.

A duração do intervalo entre as consultas de enfermagem será de acordo com a necessidade de cada cliente, considerando a sua problemática como um todo e os aspectoss quantitaticos e qualitativos dos problemas levantados.

Exames laboratoriais, como dosagem sérica de sódio, potássio, colesterol, 
triglicérides, glicemia e ácido úrico serāo solicitadas pelo menos a cada 12 me-ses, ou numa freqüência menor, de acordo com a presença de alteraçōes.

\section{III - ANÁLISE E AVALIAÇÃO DA CONSULTA DE ENFERMAGEM}

A avaliação da consulta de enfermagem é ponto relevante da assistência, pois a partir do seu resultado a enfermeira terá condições de aprimorar e redimensionar as açōes de enfermagem implantadas.

A análise dos problemas de cada hipertenso, bem como o controle dos ní-veis tensionais e o comparecimento às consultas, são dados objetivos que fornecem subsídios para verificação da efetividade da consulta de enfermagem. Ressalta-se a importância deste recurso como ação individual junto ao hipertenso, que deve estar inserido num contexto mais amplo da assistência à saúde.

PIERIN, A.M.G.; CAR, M.R. Application form.for nursing consultation to arterial hypertension's person an ambulatory care. Rev. Esc. Enf. USP, v. 26, n. 1, p. 17-32, mar. 1992.

The purpose of this article is to enfatize the nurse's role toward persons with arterial hypertension. Therefore it's introduced an application form that is used for nursing consultation to outpatients.

UNITERMS: Nursing consultation, Hypertension.

\section{REFERENCIAS BIBLIOGRÁFICAS}

1. BRASIL. Ministério da Saúde. Normas técnicas para o programa nacional de educação e controle da hipertensão arterial. Brasília, 1988.

2. CAR, M.R. et alii. Crenças de saúde do paciente com hipertensāo arterial. Rev. Paul. Enf., v. 8, n. 2, p. 4-7, 1988.

3. CHIAVERINI, R. et alii. Doença Hipertensiva São Paulo, Atheneu, 1980, cap. 1, p. 1-6: Hipertensāo arterial.

4. GIORGI, D.M.A. Estudos sobre algumas variáveis que influeciam a aderência do tratamento em hipertensāo arterial, São Paulo, 1989. 71 p./ Tese (Doutorado) - Faculdade de Medicina da Universidade de São Paulo.

5. NUSSENZVEIG, I. Hipertensão essencial. Manifestaçōes clínicas, evoluçāo e prognóstico. In: CHI-VERINI, R. et alii. Doença Hipertensiva. São Paulo, Atheneu. 1980. cap. 5, p. 117-47.

6. PAVANI, L.D.M. et alii. Consulta de Enfermagem ao cliente hipertenso: análise de um instrumento utilizado e proposta de um novo modelo. Rev. Esc. Enf., v.22, n. 1, p. 85-102, 1988.

7. PIERIN, A.M.G. A pessoa com hipertensão arterial em tratamento no ambulatório: estudo sobre os problemas, dificuldades e expectativas quanto à doença e tratamento. São Paulo, 1985. 109 p. Dissertação (Mestrado) - Escola de Enfermagem da Universidade de São Paulo.

8. RIBEIRO, A.B. Hipertensão arterial. São Paulo, Marques Saraiva, 1988.

9. SPRITZER, N. Observância ao tratamento da hipertensāo arterial. Rev. Bras. Med., v. 45, n. 10, p. 418-21, 1988. 


\section{ANEXO I}

Consulta de Enfermagem ao Cliente Hipertenso

\section{I - Identificação}

Nome:

R.G.: № Liga:

Sexo: Idade: Cor:

Escolaridade:

Orientação anterior de enfermagem Ocupação:

Sim ( )
Não ( )

Data $1^{a}$ Consulta:

\section{II - Entrevista}

1 - Explique qual é o seu problema de saúde.

2 - Sabe que tem pressão alta?

Sim ( ) Há quanto tempo?

Não ( )

3 - Toma remédios para controlar a pressão alta?

Sim ( ) Quais? Dose Horário Quem indicou?

Não ( )

4 - Utiliza outros meios para controlar a pressão alta?

Sim ( ) Quais?
Chás ( )
Homeopatia ( )
outros:

Não ( )

5 - Sentiu algo diferente, no organismo, depois que começou a tomar os remédios?

$$
\text { Sim ( ) O quê? }
$$

freqüência urinária ( ) sonolência ( ) cansaço ( ) cefaléia ( )

Não ( )

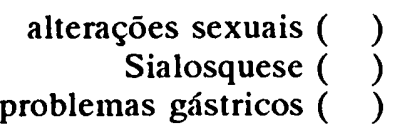
outros: 
6 - Desde o início do tratamento, deixou de tomar os remédios alguma vez?

Sim ( ) Por que?

acabaram ( )

sentiu-se mal c/ remédios ( )

a pressão normalizou-se ( )

não acredita que os remédios abaixem a pressão ( )

outros:

Não ( )

7 - Tem alguma dificuldade para obter os remédios?

Sim ( ) Quais?

Falta de dinheiro ( )

Não há na farmácia do hospital ( )

outros:

Não ( )

8 - Qual a composição básica da alimentação?

Café da manhã:

almoço:

lanche:

jantar:

outros:

9 - Módificou a quantidade de sal da alimentação?

Sim ( ) Como?

Diminuindo sal no preparo ( )

Evitando salgados, conservas, enlatados ( )

Tirou todo sal ( )

outros:

Não ( )

10 - Modificou a ingestão de alimentos que engordam?

Sim ( ) Quais?

$\begin{array}{ll}\text { Massas( ) } & \text { Doces ( ) } \\ \text { Refrigerantes ( ) } & \text { Gorduras ( ) }\end{array}$

outros:

Não ( ) 
11 - Qual a ingestão de líquidos diariamente (tipo e quantidade)? Água:

Sucos, refrigerantes:

Chá, café:

Bebida alcóolica:

Outros:

12- Qual a atividade física rotineira?

Trabalho:

Esporte:

Outros:

13 - Foi indicada, pelo médico, alguma reduçãon na atividade física?

Sim ( ) Qual

Não ( )

14 - Apresenta alteração no sono?

Sim ( ) Qual?

insônia ( ) aumento de sono ( )

outros:

Não ( )

15 - Tem o hábito de repousar durante o dia?

Sim ( ) Quando?

Não ( )

16 - Apresenta alteração no trato gastro-intestinal?

Sim ( ) Qual?

Obstipação ( ) “Queimação gástrica” ( )

Diarréia ( ) Empachamento ( )

outros:

Não ( )

17 - Apresenta alteração na eliminação urinária?

Sim ( ) Qual? (especificar)

no volume urinário ( )

nas características da urina ( ) 


$$
\text { no ato da micção ( ) }
$$

Não ( )

18 - Tabagismo

Nunca fumou ( )

Fumou anteriormente ( ) Quanto tempo? (Quantidade/dia)

Fuma atualmente ( ) Há quanto tempo? (Quantidade/dia)

19 - Uso de hormônios anticoncepcionais

Nunca usou ( )

Usou anteriormente ( ) Quanto tempo?

Usa atualmente ( ) Há quanto tempo?

20 - Conhece as complicações que a pressão alta pode trazer, se não for tratada?

$\operatorname{Sim}(\quad$ Quais?

"Derrame" ( )

Infarto ( )

Problemas renais $(\quad)$

Outros:

Não ( )

21 - Tem dificuldade em comparecer às consultas médicas e/ou para realização de exames?

Sim ( ) Qual?

Não ( )

III - Exame Físico e dados pessoais

1 - Pulso:

2 - FC:

3 - PA deitado:

em pé:

4 - Peso:

5 - Altura:

6 - Circunferência Membro superior direito:

7 - Cabeça e Pescoço:

a) boca 
b) olhos

c) ouvidos

d) nariz

e) pescoço

f) Sintomas Específicos:

$\begin{array}{ll}\text { cefaléia }(\text { ) } & \text { tontura }(\text { ) } \\ \text { edema face ( ) } & \text { dislipidemia }(\quad)\end{array}$
outros:

g) orientação auto e alopsíquica

8 - Tronco e abdômen

a) integridade do revestimento cutâneo

b) mama

c) alterações cárdio circulatórias

d) outros:

9 - Membros superiores

a) Integridade do revestimento cutâneo

b) Alterações na locomoção, sensibilidade, movimentação

c) Outros

10 - Membros inferiores

a) Integridade do revestimento cutâneo

b) Alteraçōes na locomoção, sensibilidade, movimentação

c) Sintomas específicos

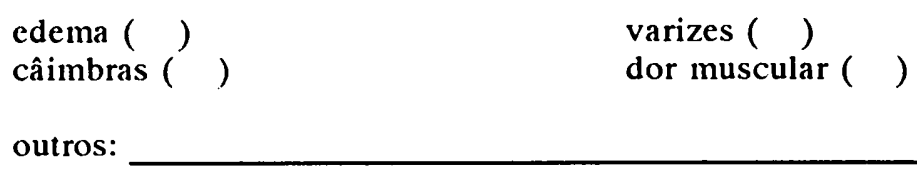


11 - Sistema reprodutor

a) uso de métodos contraceptivos

b) realização periódica de Papanicolau

c) alterações menstruais

d) alteraçōes vaginais

e) alteração na vida sexual

f) outros

\section{2- Queixas}

de ordem física

de ordem social

de ordem emocional

de ordem espiritual

13 - Imunização

14 - Dados Complementares 
Nome:

COREN 
VI - Evolução de Enfermagem

Data:

I

Nome:

COREN 


\section{ANEXO II \\ Procedimento de verificação da PA}

I - Em posição deitada

1. orientar o cliente sobre o que será feito;

2. colocar o cliente deitado no divã em decúbito dorsal horizontal (DDH);

3. deixá-lo repousar cerca de 10 a 15 minutos em ambiente tranqüilo;

4. descobrir o membro superior direito, deixando-o numa angulação de $40^{\circ}$ a $45^{\circ}$ em relação ao eixo do corpo;

5. ajustar o manguito no braço, cerca de $3 \mathrm{~cm}$ acima da fossa cubital;

obs.: certificar-se de que o manguito esteja completamente desinflado e ajustado ao braço do cliente, sendo que a bolsa inflável deverá cobrir pelo menos $2 / 3$ do braço.

6. fechar a saída de ar, apertando o parafuso da pera de borracha;

7. colocar o estetoscópio nos ouvidos, certificando-se de que a curvatura das olivas estejam voltadas para frente;

8. identificar a artéria braquial, palpando-a com os dedos indicador e médio, na porção mais interna da fossa cubital;

9. inflar o manguito até cerca de 20 a $30 \mathrm{mmHg}$ após o desaparecimento do pulso braquial;

10. colocar o diagrama do estetoscópio na artéria branquial;

11. abrir lentamente o parafuso da pera, observando a descida do ponteiro do manômetro numa velocidade de $\mathbf{2 - 4} \mathrm{mmHg}$ por segundo, inicialmente;

12. marcar a pressão sistólica ao ouvir um som claro, seguido por batidas regulares;

13. marcar a pressão diastólica considerando o desaparecimento do som;

obs.: quando houver um abafamento de som e não desaparecimento, considerar como a pressão diastólica o início do abafamento;

14. anotar os valores encontrados na ficha de consulta de enfermagem;

obs.: este procedimento deverá ser repetido 3 vezes consecutivas, considerando-se um intervalo de 1 a 2 minutos entre as mesmas, devendo-se, após, calcular a média aritmética entre os valores encontrados.

II - Repetir o procedimento acima descrito, para aferição da Pressāo Arterial, com o cliente em pé. 